\title{
Ampliación de la Iniciativa de la Cuenca del Caribe
}

\author{
René de León \\ Embojodor de El Solvodor en Woshinglon
}

$\checkmark$ racias, muy buenas tardes, quisiera agra decer a la Universidad Tecnológica, por haberme invitado a este seminario y por tener la oportunidad de compartir con ustedes algunos aspectos importantes, sobre todo lo que es la implementación de la Iniciativa cle la Cuenca del Caribe. ahora que todo el proceso político de aprobación en el gobierno de Estados Unidos ha culminado y también agradecer a la ASI, a su presidente y a su Junta Directiva por damos la oportunidad de compartir con el sector privado algunos pensamientos, algunas ideas sobre la Iniciativa de la Cuenca del Caribe y a ustedes por el interés en esta temática.

Quizás habria que comenzar diciendo que la Inicialiva de la Cuenca del Caribe es un esquema de preferencias comerciales, que es un animal político comercial muy distinto a un TLC, como el que acabamos de estar escuchando en este momento. A diferencia de lo que señalaba mi buen amigo Rigoberto Monge. la Iniciativa de la Cuenca del Caribe es temporal. es discrecional y unilateral, eso la diferencia de todo lo que puedaser un acuerdo de libre comercio donde hay concesiones reciprocas. donde hay certidumbre. donde hay permanencia donde hay predectibilidad. La iniciativa de la Cuenca del Caribe entonces, politicamente y a nivel empresarial es un animal bastante peculiar, bastante distinto a lo que es un acuerdo de libre comercio.

\section{Una exposición de la Cuenca del Caribe}

Les digo esto porque la iniciativa de la Cuenca del Caribe en realidad para los Estados Unidos, que es quien nos está otorgando obligatoriamente las preferencias, más que un esquema comercial, que si lo es, es también un esquema de polf́tica exterior de los Estados Unidos y por lo tanto se intercepta con muchos de los temas de la agenda bilateral que el país tiene con ese país en temas que no tienen nada que ver, en algunos casos con el comercio. condicionalidades de tipo político, que vienen atadas a la iniciativa del la Cuenca del Caribe a la extensión de la Cuenca del Caribe, que no son nuevas, que ya están en a'lgunos casos en la iniciativa de la Cuenca del
Caribe original, de la cual ya el país se esıá beneliciando. peroque si sonelementosque hay que tomarlos cn cuenta porque van perfilando una nueva relación no solamente económica y comercial con los Estados Unidos . sino también una nueva relación política con ese país.

Por ejemplo, el hecho de que la iniciativa de la Cuenca del Caribe tenga condicionalidades laborales. Claro, las condicionalidades laborales existen desde la iniciativa de la Cuenca del Caribe original, pero ahora el sentido de concientización de poder usar esas condicionalidades para suspender los beneficios, para revocar los beneficios, para ejercer presión en términos de cambios en la legislación laboral en El Salvador. tiene un mayor nivel de visibilidad política en el Congreso de Los Estados Unidos y en el Gobierno de los Estados Unidos es determinante. Condicionalidades en materia de propiedad intelectual, que también ya estaban en la iniciativa del la Cuenca del Caribe anterior. ahora revisten una nueva visibilidad politica

El Salvador, por ejemplo, va tener en el mes de septiembre una revisión del cumplimiento y de la aplicación de su ley de propiedad intelectual, sobre todo en lo que tiene que ver con el respeto a la propiedad intelectual de los software de computadoras y dicho sea de paso, el gobierno de los Estados Unidos el viernes pasado, aquí está una publicación en el diario oficial de Los Estados Unidos, en la cual se somete a consideración de todo el público de los Estados Unidos la elegibilidad de los paises para que gocen de esos beneficios. en la cual grupos cle presión, empresas que puedan verse perjudicadas, individuos, ONGs, grupos de la sociedad civil pueden emitir su opinión y decir que efectivamente un pais se merece o no el ser declarado beneficiado de la Iniciativa de la Cuenca del Caribe. Pero esto es una realidad que es inherente a una concesión de tipo unilateral, yo estoy claro que en este mismo lugar en este mismo auditorio, que nos encontramos en este momento, se podrán recordar del acuerdo de alcance parcial con México y de las innumerables discusiones que se han tenido acá, sobre ese acuerdo de alcance parcial, que era una preferencia unilateral de México y que ahora se ha visto de alguna manera superada la concesión unilateral de México a través de un acuerdo de libre comercio. 
Ampliación de la Iniciativa de la Cuenca del Caribe

ampliación de I a iniciativa de la cuenca del caribe

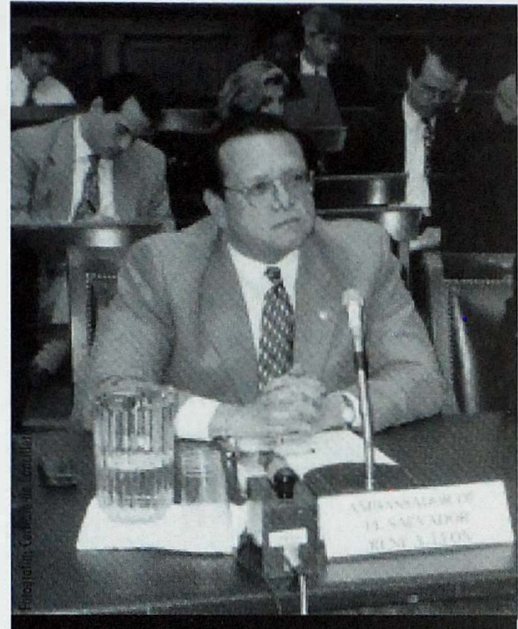

René de León, Embajador de El Solvador en Washintong.

En esa misma situación está la Iniciativa de la Cuenca del Caribe y por lo tanto hay que hacer una reflexión. no podemos ver la Iniciativa de la Cuenca del Caribe fuera de las discusiones de respeto de los derechos laborales en nuestro pais, no la podemos ver aisladamente de lo que es las presiones para que El Salvador legalice. por ejemplo, a través de sus empresas que ocupan software. la situación de la piratería de programas de computación, no la podemos ver separadamente de una serie de aspectos que ustedes, se podrian poner a pensar que no tienen nada que ver con el ámbito comercial, como podría ser el tratamiento no discriminatorio de manera transparente de las compras gubernamentales de una empresa de Los Estados Unidos, participando en una licitación én El Salvador, que de alguna manera están amarradas a mantener el estátus de pais beneficiado de la Iniciativa de la Cuenca del Caribe.

$\mathrm{Ni}$ siquiera la lucha contra las drogas puede verse aisladamente del tema de la Iniciativa de la Cuenca del Caribe. los paises beneficiados con la iniciativa de la Cuenca del Caribe tienen que cumplir con todos los requisitos de certificación que los Estados Unidos hacen anualmente ante el proceso de evaluación de la manera como los paises de América Latina contribuyen con los Estados unidos en la lucha contra el narcotráfico, por e jemplo.

Entonces hay una intersección bien grande entre aspectos políticos propios de la relación bilateral de $\mathrm{El}$ Salvador con los Estados Unidos que están atados de alguna u otra manera a ese usufructo de esos beneficios de parte de los paises de la región. Por lo tanto, en el marco de la politica internacional de EI Salvador la I niciativa de la Cuenca del Caribe ha ocupado porque ha determinado algunas decisiones y seguirá ocupando un papel bien importante. más allá del ámbito de lo que son las especilicaciones de los benelicios que esto puede traer para la economía nacional.

Habiendo dicho eso y teniendo bien claro el concepto politico en yue debemos de ubicar la iniciativa de la Cuenca del Caribe. dentro de lo que es la integralidad de las relaciones políticas y económicas que EI Salvador mantiene con los Estados Unidos, es importante también reconocer que esta iniciativa tiene un potencial de beneficios económicos muy grandes para El Salvador por el tipo de operaciones que permiten. ahora que se realicen. para poder exportar los productos salvadorenos al mercado más grande del mundo.

Obviando los detalles técnicos quizás de la legislación, es decir cuáles son las operaciones textiles y de con「ección que pueden soliciıar, voy a de jar eso para Alfredo Milián. que dicho sea cle paso sabe más de eso que yo y que él explique los programas ocho cero siete, el programa ocho ccro nueve. las regulaciones y todas las operaciones que califican. Lo que sí puedo decirles es que la Iniciativa de la Cuenca del Caribe a través de todas estas disposiciones respalda condiciones de competitividad que la industria de conlección en El Salvador, sobre todo. habia perdido y que habia hecho que de tasas de crecimiento superiores al $50 \% 1990$ y 1997 . Ia industria de la confección y por lo tanto las inversiones asociadas a la misma hayan disminuido las tasas de crecimiento cercanas al seis por ciento en los últimos dos años. Por lo tanto la industria de la confección en El Salvador había llegado a un estado estacionario en el cuál habia dado todo lo que podia dar en términos de crecimiento económico. de generación de divisas y de generación de empleo.

\section{La creación de nuevos empleos}

En ese sentido las disposiciones de la Iniciativa de la Cuenca del Caribe. tienen un potencial de generar en El Salvador en los próximos tres años hasta ciento cincuenta mil nuevos empleos, debido a lodasesas flexibilidades de las cuales ya les va a hablar Alfredo.

Tiene también un potencial de generar una demanda por techo industrial superior a un millón de metros cuadrados, tiene un potencial de atraer hasta setecientos cincuenta millones de dólares en nuevas inversiones y adicionalmente hasta triplicar el valor agregado de la industria de la confección en nuestro pais. La industria de la confección en nuestro pais, según estimaciones de los mismos Estados Unidos, genera aproximadamente los mil trescientos millones de dólares que El Salvador está exportando en este momento en productos de confección específicos a los Estados Unidos, más o menos cua- 


\author{
Universidad Tecnológica de El Salvador
}

ampliación de la iniciativa de la cuenca del caribe

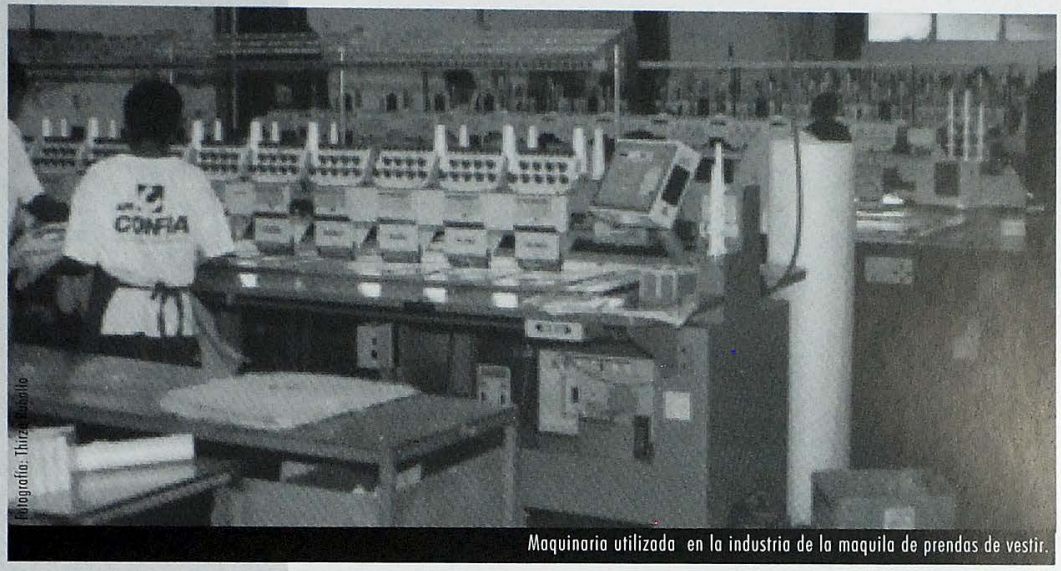

trocientos treinta y dos millones de dólares son valor agregado para El Salvador.

Claro, une hace un e jercicio muy estricto cle contabilidad maoional ouando habla de la maquila, perola verdad que en las cuentas naoionales, tradicionalmente en las exportaciones, se registiran al valor de la exportación FOB, nuncarnadie hace un ejercicio cle cuál es el valor agregado de las exportaciones, con la cual lo que se quiere decir es que esos cuatrocientos treinta y dos millones de dólares de valor agregado del sector maquila en E! Salvador reallmente pueden superar a los generados por las exportaciones de muchos productos tradicionales $y$ no tradicionales en DI Salvador. por el simple hecho que nosotros tenemos una base textil y tenemos una integraoión en paquetes completos que va desde el hilo pasando por la tela, hasta la exportación de la prenda final.

Quieroademás decirles que si eso se concreta, si el valor agregado de la industria textil en EI Salvador llega a triplicarse, p.or primera vez en la aclividad económica en nuestro país podria generarse un monto de ingresos en materia de divisas similar al de las remesas familiares, de quizás más đel $20 \%$ de la población salvadoreña que r.eside en este momentoen Los Estados Unidos.

Para maximizar y materializar los beneficios de la legislacion el gobierino de El Salvador ha presentado y ha discutido con el sector privado un programa de aprovechamiento de legislaciôn $C D I$, de la cual la señora Viceministra va a ser una exposición muy detallada y que dicho sea de paso puede ser un programa de aprovechamiento a la legislación CDI, que incluso en distintos aspeotos podría utilizarse paria un programa de atracción de nuevas inversiones en otros sectores que no necesariamente sean el sector textil.

Deseo destacar lambién que la lćgislación CDI licne algunas disposiciones especificas para sectores sobrc todo de pequeña empresa y de mediana empresa en algunas actividades como es la producción de calzado, en la cual también se abren oportunidades que no necesa riamente estaban disponibles para empresarios de la economia salvadorena, al darle el mismo tratamiento del NAFTA, a algunos productos que formalmente estaban excluidos cle la legislación CDI, predominantemente la exportación de atün enlatado, las exportaciones cle calzado, que solamente se permitian del 100\% de maquila de calzado con complementos de los Estados Unidos. ahora se le da el mismo tratamiento del NAFTA al calzado salvadoreño y también en lo que son las indusirias periféricas que rodean a la industria de la maquila como son por ejemplo todo lo que es las industrias de empaques y todo lo que son las industrias de tratamiento de telas y Iratamientos cle ropa. una vez producidas esies productos.

Esıo ya esıá generando una reacción económica muy positiva en El Salvador y personalmente puedo testimoniar de que proyectos que estaban siendo retrasados de inversiones en zonas francas, proyectos que estaban siendo retrasados por la falta de paridad en el CDI. como podrian ser expansiones de empresas que ya se encuenIran haciendo negocios en EI Salvador y otros nuevos proyectos que su faclibilidad económica dependia de que esta legislación fuera aprobada ya esıán caminando y ya estamos viendo algunas empresas que están ejeculando esos proyectos de expansión y sobre todo, es que contratos que se habian perdido por la desviación de comercio y la desviación de inversiones al estar el NAFTA estacionado en la industria de maquila nacional. Restituirse esas condiciones preNAFTA, también han generado una gran actividad de empresas extranjeras nuevas que quieren nuevamente volver a realizar negocios con el pais. Tenemos también empresas y esa es una de los principales objetivos de tener en cuenta en la Iniciativa cle la Cuenca del Caribe. tenemos empresas que en este momento estaban haciendo sus contactos fuera de la región CDI y lo estaban haciendo en Asia. Están moviendo sus contratos de Asia, están moviendo sus contraıos de Arabia Saudita, están moviéndose de Perú. que están 
moviendo sus contratos de Turquia y están hablando con empresas nacionales para que estas empresas puedan asumir esos contratos y esa producción ahorahecha desde EI Salvador goce del tratamiento del libre comencio y del tratamiento de libre cuota.

\section{Intereses norteamericanos}

Estamos en este momento trabajando en todo lo que es el proceso de implementación de esta legislación, quiero decirles que no es fácil, hay muchas áreas grises y que es la implementación de la legislación CDI, sobre todo porque el proceso de la aprobación del CDI, responde más a un proceso político interno de los Estaclos Unidos a una negociación de los intereses de los Estados Unidos entre la Casa de Representantes y el Senado y por lo tanto hay todavia algunas disposiciones que fueron añadidas a última hora y que todavia no conocemos los verda deros alcances de esas disposiciones y solamente se van a definir al momento de tener lista la reglamentación, para la aplicación de la legislación CDI, y con el Ministerio de Economia y el sectorprivadohemos estado trabajando, no solamente en la definición de la cuota, tanto de tela regional y de tela salvadoreiia, para la producción cle estas prendas, sino que también en la otra cuota de cuatro punto dos millones cle docenas para las exportaciones de camisetas y en otros temas que van a maximizar el valor económico que la legislación CDI puede dejar para El Salvador.

Yo quisiera terminar mi intervención diciendo también que la legislación CDI va a definir un nuevo marco de política comercial en El Salvador y de negociaciones comerciales internacionales, por varias razones, porque tampoco la legislación CDI se puede ver aisladamente de los plazos que establece el acuerdo de textiles y vestuarios en la Organización Mundial del Comercio para la plena liberalización del comerciotextiles a partir del anoo dos mil cinco. La legislación CDI Iampoco puede verse aisladamente de las negociaciones bilatcrales que los Estados Unidos ha tenido con China Comunista, para cl ingreso de China Comunista a la Organización Mundial del Comercio, que también van afectar la manera en que el CDI puede rendir resultados cconómicos en nuestro pais.

\section{EE UU el mejor socio comercial}

Tampoco puede verse independientemente el proceso de formación de Área de Libre Comercio de las Américas, porque esa es la prioridad comercial de Los Estados Unidos en las economías emergentes de América Latina. Tener una zona de libre comercio funcionando a partir del año dos mil cinco, en las que participen las treinta y cuatro democracias de estc hemislerio y por lo tanto para los Estados Unidos la legislación CDI es una pie dra de toque, para la concreción del alta y finalmente tampoco podemos ver esta legislación fuera del mandato que el gobierno de Estados Unidos, el mandato, insisto en el mandato que el Congreso de los Estados Unidos le da al Presidente de los Estados Unidos de negociar un acuerdo de libre comercio entre los paises beneficiados dcl CDI o regiones del CDI y los Estados Unidos.

En pocas palabras, la legislación CDI nos pone en la agenda comercial de EI Salvador y $\mathrm{cl}$ ingreso de China a la OMC, nos pone forzosamente a prestarle atención a la integración de los textiles en la OMC, en el dos mil cinco, nos obliga a vincular esa legislación con el avance del Libre Comercio en las Américas y a negociar o buscar los mecanismos para la eventual negociación y activar los mecanismos en algunos de los que ya existen de un acuerdo de libre comercio con los Estados Unidos, que dicho sea de paso es el principal socio comercial de la región, visto cada país individualmente, algunos de la región centroamericana y de toda la región en su conjunto.

Esto es sumamente importante, porque a diferencia también del acuerdo económico, no solamente no es lo mismo establecer un acuerdo de libre comeicio con los Estados Unidos, que un acuerdo de libre comercio con México, sino que además un acuerdo de libre comercio con tos Estados Unidos conlleva la negociación de un acuerdo de libre comercio con el principal socio comercial, con una economia complementaria y con una economía con la cual a nivel de importaciones y exportaciones toma socios comerciales más importantes que la misma China continental, que los mismospaises de la Unión Soviética, que el mismo Brasil, que el mismo Chile oque la misma Rusia en este momento o que la India y por lo tanto si puede significar la posibilidad de negociar un acuerdo de libre comercio con los Estados Unidos un cambio drástico en lo que es no solamente la política comercial, sino que el comportamiento de las grandes variables de la balanza de pagos de nuestro país y un empuje con un acuerdocrecimiento que no tiene paralelo en materia de generación de empleo, de generación de inversiones y en materia de actividades de complementación económica entre la economía más desarrollada del mundo y nuestro pais.

Yo me quisiera quedar hasta acá para ahondar en los temas que ustedes consideren más importantes y específicamente en el área de preguntas y respuestas trabajar un poquito más, perfilar un poquito más lo que sería incluso el seguimiento a las negociaciones de libre comercio que en este momento está teniendo Costa Rica con Canadá. Porque también esas negociaciones de libre comercio van a tener mucho que decir en una eventual negociación de un acuerdo de libre comercio con los Estados Unidos, el mecanismo existe, es un foro regional de los países centroamericano con los Estados Unidos que empezo a funcionar en 1998. Yo me recuerdo que la primera vez que lo dije a un presidente de los Estados Unidos en una presentación que hice en San José. Costa Rica, en Marzo de 1997 que tenia sentido economico para Estados Unidos y para la región centroamericana negociar un acuerdo de libre comercio, los asesores del presidente Clinton me vieron como si era marciano, y esıe tipo de que está hablando, en que basa, cuál es el fundamento de poder convencer a una economía como los Estados Unidos de negociar un Acuerdo de Libre comercio con cinco paisitos de Centroamérica y creo que ahora la legislación CDI solamente puede tener sentido de largo plazo, para generar los mismos beneficios de un acuerdo de libre comercio, si la sabemos utilizar para iniciar un acuerdo de libre comercio con quien verdaderamente necesitamos uno de manera urgente, sería Estados Unidos. 